\title{
Brain Imaging and Hepatic Encephalopathy
}

\author{
Neeral R Patel BSc, "Mark JW McPhail PhD MRCP, \\ Simon D Taylor-Robinson MD FRCP
}

Liver and Antiviral Center, Department of Medicine, St Mary's Hospital Campus, Imperial College London, London, United Kingdom

\author{
*Address for correspondence: \\ Dr Mark JW McPhail \\ Liver Unit, Department of Medicine, $10^{\text {th }}$ Floor QEQM Wing, St Mary's Hospital Campus, \\ Imperial College London, South Wharf Street, London, W2 1NY, United Kingdom \\ Tel: +44 2078866454 \\ Fax: +442077249369 \\ Email: mark.mcphail@imperial.ac.uk \\ Mr Neeral Patel \\ Liver Unit, Department of Medicine, $10^{\text {th }}$ Floor QEQM Wing, St Mary's Hospital Campus, \\ Imperial College London, South Wharf Street, London, W2 1NY, United Kingdom \\ Tel: +442078866454 \\ Fax: +44 2077249369 \\ Email: neeral.patel06@imperial.ac.uk \\ Professor Simon D Taylor-Robinson \\ Liver Unit, Department of Medicine, $10^{\text {th }}$ Floor QEQM Wing, St Mary's Hospital Campus, \\ Imperial College London, South Wharf Street, London, W2 1NY, United Kingdom \\ Tel: +44207886 6454 \\ Fax: +44 2077249369 \\ Email: s.taylor-robinson@imperial.ac.uk
}

\section{KEY WORDS}

Hepatic encephalopathy; Brain imaging; Magnetic resonance imaging; Magnetic resonance spectroscopy, Computed tomography, Single photon emission computed tomography; Positron emission tomography. 


\section{SYNOPSIS}

Novel imaging techniques are allowing hepatologists to investigate the structural and functional neuropathology of hepatic encephalopathy $(\mathrm{HE})$ in greater detail but only limited techniques are applicable to the clinic. Computed tomography and magnetic resonance imaging (MRI) can rule out other diagnoses and in the case of MRI, give certain key diagnostic features in widely available sequences. While increased brain water content is a hallmark of HE, the localisation of low-grade cerebral edema, the extent of regional swelling or atrophy and the different functional characteristics of affected brain regions continue to be debated. More specialised volumetric, diffusion-tensor, magnetization transfer, functional magnetic resonance imaging and magnetic resonance spectroscopy, in conjunction with positron emission tomography continue to enrich the investigative findings in HE. Nevertheless an internationally accepted diagnostic framework that includes an objective imaging test to replace or augment psychometry remains elusive. Quantitative MRI is likely to be the best candidate to become such a test and the utility of MR and nuclear medical techniques to the clinic and results from recent research are described in this article. 


\section{Introduction}

Hepatic encephalopathy (HE) is a neuropsychiatric disturbance affecting patients with acute liver failure (ALF), cirrhosis or non-cirrhotic portosystemic bypass (1). The clinical spectrum of HE extends from mild cognitive impairment, to coma and death (2). The majority of cases involve patients with minimal HE (MHE), which has been associated with an impaired ability to drive automobiles safely, reduced health-related quality of life and increased risk of hospitalization due to overt HE (3-5).

The disease process is multifactorial, with hyper-ammonaemia, gut-derived toxins, short and medium chain fatty acids, cerebral manganese deposition and relative deficiencies in circulating amino acids with consequent neurotransmitter imbalance commonly implicated. There is consensus that ammonia is central to the pathogenesis (6). Varying degrees of cerebral edema may result from the uptake of excess ammonia into astrocytes, with subsequent conversion to glutamine, which acts as a cerebral osmolyte, mitochondrial toxin and instigator of neurotransmitter instability (7-11).

Currently, the diagnosis of $\mathrm{HE}$ lacks standardization, particularly in $\mathrm{MHE}$, which requires neuropsychiatric testing to detect cognitive impairment. Psychometric test results can be dependent on the patient's age, educational status, emotional affect and linguistic abilities. Evaluation varies between countries while in some forms of testing, considerable expertise and facilities to conduct an assessment are required (12). This perceived diagnostic difficulty leads to under recognition of this important clinical problem. Even when the presentation of HE is clinically overt, the grading and assessment of longitudinal change is subjective among clinicians, and is also prone to disagreement or even misdiagnosis (12).

The cerebral insults secondary to hepatocellular failure or portosystemic shunting result in structural and functional abnormalities in the brain, which imaging may detect and quantify. Suitable modalities include magnetic resonance imaging (MRI), magnetic resonance spectroscopy (MRS), computed tomography (CT) in conjunction with positron emission tomography (PET) and single photon emission computed tomography (SPECT) (Table 1 and Box 1). These imaging techniques may allow the development of tools for the objective, reproducible and non-invasive diagnosis and monitoring of HE. 
While stand-alone computed tomography (CT) is useful for determining gross structural lesions such as cerebral edema in ALF or other pathologies in patients with CLD, it is limited as a diagnostic and longitudinal research tool, owing to poor sensitivity and repeated exposure to ionizing radiation. MRI and PET/SPECT will thus be the focus for this article with particular focus on patients with HE secondary to cirrhosis, where most possibility for diagnostic doubt or pathophysiological disagreement exists.

\section{Magnetic Resonance Imaging}

$\mathrm{MRI}$ is currently the most frequently utilized imaging tool in HE research studies and even standard clinical sequences can give information supporting a diagnosis of HE. A clinical set of sequences when requesting an "MR Brain" study would usually consist of $T_{1}$ - and $T_{2}$-weighted sequences as standard and a selection of more advanced $\mathrm{T}_{2}$-weighted sequences such as Fluid Attenuated Inversion Recovery (FLAIR) or Diffusion-Weighted Imaging (DWI).

\section{1 $\mathrm{T}_{1}$-weighted MRI}

Bilateral and symmetric hyper-intensity of the basal ganglia, using $T_{1}$-weighted MRI sequences in patients with cirrhosis is an observation with some clinical utility (Figure 1) (14-17). It has been postulated that excess circulating manganese is the cause of such hyperintensity, due to reduced hepatobiliary excretion as a result of liver failure, and subsequent deposition in the basal ganglia where blood flux is high $(18,19)$. This theory has been corroborated by the correlation between blood and cerebrospinal fluid manganese levels with $\mathrm{T}_{1}$-weighted hyperintensity; and also the normalization of basal ganglia hyperintensity and blood levels of manganese after liver transplantation (20-22).

However, the results of studies investigating the relationship between manganese-related pallidal hyperintensity and psychometric performance in HE are conflicting $(23,24)$. It is therefore unclear whether $\mathrm{T}_{1}$-weighted hyperintensity represents a manifestation of $\mathrm{HE}$, or if it is an occurrence secondary to cirrhosis, cholestasis and/or porto-systemic shunting $(25,26)$. The preferential deposition of manganese in the basal ganglia has been suggested to be an explanation for the Parkinsonian-like symptoms, which can arise as a result of HE (27). So while a useful adjunct this 
lack of correlation with grades of $\mathrm{HE}$ makes a single $\mathrm{T}_{1}$-weighted exam insufficient in MR Brain in patients with cirrhosis.

\section{2 $\mathrm{T}_{2}$-weighted MRI: Fast FLAIR Imaging}

Fast FLAIR (Fluid Attenuated Inversion Recovery) $\mathrm{T}_{2}$-weighted imaging represents a MRI sequence that has been shown to be sensitive for the detection of diffuse high intensity white matter lesions (WMLs) $(28,29)$. WMLs may develop secondary to cerebrovascular small-vessel disease, which neuropathologically, are a combination of reversible edema and irreversible neuronal damage. (30). Minguez and colleagues noted WMLs were found to be reduced in volume and number after treatment with neomycin and/or branched-chain amino acids in conjunction with improved psychometric performance (31). To further investigate this observation, the same investigators compared WML volumes in patients with impaired cognition, pre- and post-liver transplantation (32). A significant reduction in WML volume was detected after liver transplantation (Figure 2), as well as a strong negative correlation between fast FLAIR $\mathrm{T}_{2}$-weighted lesion load and psychometric score. Rovira and colleagues, and Cordoba and colleagues, measured high-signal intensity, along the corticospinal tract on fast FLAIR $\mathrm{T}_{2}$-weighted images in patients with chronic liver disease, with subsequent signal normalization after liver transplantation. $(33,34)$.

\section{3 $\mathrm{T}_{2}$-weighted MRI: Diffusion Weighted Imaging}

Diffusion-Weighted MRI (DW-MRI) is a commonly available sequence often used in assessing neurovascular disease which assesses changes in the motion of water molecules, by defining the chemical interaction between water and cellular barriers (35). The diffusivity of water molecules can be quantified by calculating the apparent diffusion coefficient (ADC) and can distinguish between vasogenic (interstitial) and cytotoxic edema $(36,37)$.

Several studies have consistently demonstrated a link between increased cerebral ADC (demonstrating interstitial edema) and patients with either MHE or overt HE; the degree of brain water diffusivity also correlating with grade of HE (37-40). DW-MRI studies have countered the traditional astrocytic swelling hypothesis, suggesting increased ADC values relate to purely extracellular water accumulation in low-grade cerebral edema, secondary to chronic HE $(37,41-43)$. Theories that explain 
this phenomenon include hyperammonemia-induced increased blood-brain barrier permeability and reduced glial fibrilliary acidic protein (GFAP) expression, a protein that regulates astrocytic permeability; reduced membranous GFAP in astrocytes has been previously linked increased diffusivity in the extracellular space (44-46). Discrepancies in studies where ADCs have been raised or lowered in clinically defined HE may be related to the onset of disease. Lower ADC values have been noted in patients with HE, secondary to ALF (and cytotoxic edema), whereas higher periventricular white matter and basal ganglia ADC in patients with $\mathrm{CHE}$ have been suggested to support a finding of interstitial edema (47).

Detection of water diffusion has improved in recent years with the development of diffusion tensor MRI (DT-MRI), a technique that provides detailed information on brain tissue structure. Fractional anisotropy (FA, where the fractional free or bound water component is estimated) maps can be assimilated to perform "tractography", a method of determining underlying brain anatomy and where edema is localised (48). Kumar and colleagues demonstrated increased mean diffusivity in the internal capsule and cortical gray and white matter of HE patients, but differential patterns of correlation between mean diffusivity and fractional anisotropy in the corpus collusum suggested that interstitial edema was the primary HE correlate in this study of 14 low-grade HE patients. While DWI is often used in clinical scanners, and we would recommend using $T_{1}$-weighted and FLAIR/DWI $T_{2}$ weighted sequences, the statistical requirements of DTI make it currently unsuitable for standard clinical use.

\subsection{Volumetric MRI}

Shah and colleagues correlated quantitative $T_{1}$ mapping of cerebral water content in the putamen, globus pallidus and occipital white matter with severity of HE (13). This was one of the first direct and quantitative measurements of increased regional cerebral water content with more severe grades of $\mathrm{HE}$, but the imaging technique is highly specialised. Cerebral edema that occurs as a result of ALF can be routinely viewed using MRI (49), but is often low grade and is hence undetectable by radiologists in chronic HE. The question arises of whether a simple measure of total brain volume (BV) would be useful. Recent developments in software packages for brain volumetry allow small $(<1 \%$ total $\mathrm{BV})$ changes in brain size in $\mathrm{HE}$ to be quantitated, allowing precise measurements of $\mathrm{BV}$ 
(50) on relatively standard $T_{1}$-weighted MR sequences (51-55). Single time-point determination of brain volume is considerably less accurate than when BV change is determined longitudinally, owing to the ability to co-register (align) images using the theoretically unchanged skull surface, thus allowing more robust determination of CSF and gray and white matter densities $(50,56)$. Further structural information can be determined using voxel-based morphometry (VBM) where regional contribution to brain volume change can be calculated; this has been applied in many neuropathological scenarios (57).

A pilot study conducted by Patel and colleagues, was the first to utilize co-registered MRI techniques to determine changes in BV in chronic liver disease (58). This small scale investigation focused on six patients with MHE, and three patients who had been diagnosed with overt HE. The authors concluded that in patients treated with lactulose brain volume fell, in association with improved psychometric performance.

In contrast patients with HE often have risk factors for cerebral atrophy (such as age, alcohol abuse and possibly cirrhosis itself) and these effects couldalso contribute to neuropsychological impairment and apposite structural brain changes. Garcia-Martinez and colleagues investigated cognitive function, cerebral magnetic resonance spectroscopy and BV post-liver transplantation (59). Posttransplantation, BV was reduced in patients with prior HE, correlating with worse neuropsychiatric score in this group. This study, suggests that brain atrophy accrued prior to liver transplantation, but masked by low grade edema, may also play a role in cognitive dysfunction post-transplantation. Further evidence of the contribution of atrophy in patients with hepatic encephalopathy has recently emerged with the identification areas of atrophy throughout the cortex and white matter quantitated by VBM and correlating with the severity of encephalopathy (60). A lack of corroborative MR spectroscopy or more advanced water localisation sequences could not add further mechanistic data to this interesting observation, which requires validation from other groups.

\subsection{Magnetization Transfer Imaging}

Magnetization transfer imaging (MTI) improves image contrast as a consequence of the magnetic properties of free and bound protons (61). Free protons are present in water molecules, whereas 
bound protons are fixed to macromolecules, such as proteins, lipids, carbohydrates and nucleic acids (62). Magnetization transfer between bound and free protons reduces the signal intensity observed in the resultant MR image $(17,25)$. MTI also allows for the magnetic transfer ratio (MTR) to be quantified, which ultimately reflects brain parenchymal changes: a low MTR indicates neuronal damage as well as an increase in water content or membrane permeability $(17,63)$.

Lower MTRs have been demonstrated in patients with HE, including a cohort who developed MHE secondary to extra-hepatic portal vein obstruction (64-66). A study by Cordoba and colleagues verified this by concluding MTR normalisation post-transplantation reflected the correction of lowgrade cerebral edema in patients with $\mathrm{MHE}$, and that the basal ganglia and white matter are initial targets for water accumulation (67). A study by Miese and colleagues that utilized both MTI and DWMRI to investigate cerebral edema in HE, found that both reduced MTR and raised ADC were correlated with HE grade in non-alcoholic patients (40). However, in patients continuing to drink to alcohol to excess, no such correlation was established, suggesting chronic alcohol mis-use may independently cause cerebral oxidative damage in this group, although as has been noted above, the effect of atrophy on these measurements may be underestimated (68).

\subsection{Functional MRI}

Functional MRI (fMRI) measures changes in deoxyhaemoglobin concentration (a substance with paramagnetic properties, relative to tissue) that occurs as a result of the rise in blood oxygenation during neuronal activity (69). The subsequent blood oxygen level dependent (BOLD) contrast highlights areas of activity in the brain.

fMRI has the benefit of being a non-invasive and safe investigatory tool in low grade HE (as these patients can follow instructions in the scanner), that is particularly useful in longitudinal studies as no radioactive marker injections are required (35). Zafiris and colleagues demonstrated that MHE is associated with impaired coupling between visual judgment areas (70). A study conducted by Zhang and colleagues compared brain fMRI data in 14 patients with cirrhosis and 14 healthy volunteers $(71)$. An incongruous word reading task and incongruous color-naming task (testing for attention and interference) highlighted various cerebral areas on fMRI: there was greater activation of the bilateral 
parietal and prefrontal cortices in the patients with cirrhosis (72). In a separate study, Zhang and colleagues concluded there was reduced functional connectivity in the right middle frontal gyrus and left posterior cingulate cortex, (part of the default-mode network [DMN)).. This highly interconnected, metabolically active and well described area of the brain is vital for the preservation of attention and is worthy of further study into whether this abnormal activation is related to hyperammonemia or lowgrade cerebral edema (73-75).

\subsection{Magnetic Resonance Spectroscopy}

MRS has been utilised in HE investigations since the 1980s, although it has been more widely used in recent times as a consequence of improved sequence development and higher field strength to resolve metabolite signals. MRS also allows for the investigation of HE at the molecular level, by studying cerebral tissue in vivo in whole-body clinical magnets (typically at 1.5-3.0T) (76).

Various nuclei can be used to determine metabolite changes in $\mathrm{HE}$, the most common clinically-used being proton $\left({ }^{1} \mathrm{H}\right)$ and 31 -phosphorus $\left({ }^{31} \mathrm{P}\right)$ MRS. ${ }^{1} \mathrm{H}$ MRS allows for the quantification of metabolites such as choline (Cho), creatine ( $\mathrm{Cr}$ ), $\mathrm{N}$-acetyl aspartate (NAA), glutamine (Gln) and glutamate (Glu) or the unresolved combination (Glx), as well as osmolytes such as myo-inositol $(\mathrm{ml})$ and taurine. ${ }^{31} \mathrm{P} \mathrm{MR}$ spectra allow definition of phosphomonoester (PME), inorganic phosphate (Pi), phosphodiester (PDE), phosphocreatine, YATP, aATP and $\beta A T P$ resonances (or also more correctly termed nucleoside triphosphate resonances [NTP], as they also contain contributions from cytosine triphosphate, guanosine triphosphate and uridine triphosphate, in addition to the overwhelming proportion from adenosine triphosphate, [ATP]) (77). These resonances provide information on cell membrane turnover with cell membrane precursors measured in the PME resonance and cell membrane degradation products measured in the PDE resonance, while information on high energy phosphate metabolism and intracellular $\mathrm{pH}$ is available from the $\mathrm{Pi}, \mathrm{PCr}$ and NTP resonances $(78-80)$. Ammonia is not detected by MRS due to rapid interchange with water (81).

The characteristic spectral appearance of $\mathrm{HE}$ on in vivo ${ }^{1} \mathrm{H}$ MRS adds further evidence to the astrocyte swelling hypothesis demonstrated by a reduction in intracellular $\mathrm{ml}$ and Cho, and a concurrent increase in Gln and Glu (Figure 3) $(77,82)$. Furthermore, these characteristic metabolic 
changes have been shown to correlate with psychometric performance (23). This suggests that cells expel osmolytes, such as taurine, $\mathrm{ml}$ and $\mathrm{Gln}$, in the face of an osmotic water load. In vivo ${ }^{1} \mathrm{H}$ MRS allows the degree of intracellular osmolyte homeostasis to be detected and monitored sequentially and to give an indirect indication of cell swelling in a non-invasive way. The technique is also open to following response to therapeutic intervention. Recent sequence development using 2-dimensional spectroscopy allows improved resolution of 1 -dimensional ${ }^{1} \mathrm{H}$ MRS and resolution of glutamine and glutamate (Figure 4) (83).

The interpretation of ${ }^{31} \mathrm{P}$ MRS is complex in vivo, since many of the resonances are multi-component and are not easily separated into their constituents at clinically-used magnetic field strengths. Overall, however, changes do appear to reflect alterations in bioenergetic pathways, and glucose utilisation (since sugar phosphates also contribute to both the PME and PDE resonances), as well as giving an indication of phospholipid membrane synthesis and degradation and of cell membrane fluidity $(77,84)$. Consensus on ${ }^{31} \mathrm{P}$ MRS studies has been hampered by small study sample sizes and inconsistencies in MRS protocols between centers (35). Studies conducted by Taylor-Robinson and colleagues have demonstrated reductions in the PME/ $\beta$ ATP and PDE/ $\beta$ ATP which correlate with reduced choline, as verified by parallel ${ }^{1} \mathrm{H}$ MRS on the same subjects $(77,84,85)$. These changes were thought to represent reduced glucose utilisation in $\mathrm{HE}$, as components of the glycolytic pathway contribute to the

${ }^{31} \mathrm{P}$ MRS spectrum in vivo. However, a change in membrane fluidity is equally possible. ${ }^{1} \mathrm{H}$ in vivo MRS is more likely to be developed for clinical use but normal ranges and diagnostic thresholds for key metabolites are not yet agreed.

\section{Nuclear medicine: Positron Emission Tomography and Single Photon Emission Computed Tomography}

PET involves the measurement of the concentration of positron emitting radioisotopes from the body. Co-registered CT or MR tomographic imaging provides structural information, while, depending on the radioligand used, functional data are available in the form of glucose and oxygen metabolism, blood flow, amino acid metabolism and rates of amino acid incorporation into proteins, acid-base balance and membrane transport (86). Positron-emitting radionuclides that are used in PET include: ${ }^{11} \mathrm{C},{ }^{18} \mathrm{~F}$, ${ }^{15} \mathrm{O}$, and ${ }^{13} \mathrm{~N}(87)$. 
Lockwood and colleagues have used ${ }^{18} \mathrm{~F}$-fluorodeoxyglucose (FDG) PET to investigate functional changes in chronic HE $(88,89)$. They demonstrated a reduction in glucose metabolism in the anterior cingulate gyrus, which may reflect the attention deficit found in many HE patients on neuropsychometric testing and in fMRI studies. Alterations in cerebral blood flow (CBF) have also been established using FDG PET and ${ }^{15} \mathrm{O}$ PET, where poor neuropsychiatric performance correlated with reduced blood flow in all cortical areas; temporal lobe CBF was found to be most discriminatory between HE patients and healthy volunteers (88).

Brain imaging with ${ }^{13} \mathrm{~N}$-ammonia, has been used to assess cerebral ammonia metabolism in (i) healthy subjects, (ii) subjects with mild liver disease, but with no evidence of cirrhosis, (iii) subjects with cirrhosis with and without $\mathrm{HE}$, and (iv) subjects with malignant neoplasms with metastases in the liver (90-94). However, the results of these studies are somewhat conflicting, particularly with respect to interpretation of blood-brain barrier (BBB) permeability in HE. This is probably owing to differences between research groups in the tracer kinetic modeling approach used to determine parameters of cerebral ammonia metabolism quantitatively. Different expert opinions exist over which conclusions should be drawn out of conflicting results in PET studies of ammonia metabolism and BBB permeability. Some proponents suggest that further studies, including larger numbers of patients and using standardized analysis techniques are necessary, in order to provide consensus and easy methodology to clarify the relationship between BBB permeability and ammonia toxicity (95).

Changes in different neurotransmission systems have been demonstrated in HE patients using radiotracer methods. Increased benzodiazepine receptor binding, decreased dopamine receptor binding and decreased binding to serotonin transporters have been shown (Figure 5) (96). These changes correspond to symptoms observed in HE (depression of neuropsychological function and extrapyramidal symptoms) and suggest possible targets for treatment (97-99). Studies of neuronal activity have been used to shed light on the pathophysiology of HE, while imaging of a potentially crucial process in HE, neuroinflammation, using the radioligand C-11-PK11195 to detect peripheral benzodiazepine receptors (PTBR), which are present on a number of cell types, including activated microglial cells, is gaining increased attention (100-102). 
Molecular imaging using SPECT requires a molecular marker that is labelled with a radionuclide, which results in the emission of gamma ray photons or high-energy X-ray photons (103). Cerebral blood flow is most commonly assessed by using ${ }^{99} \mathrm{Tc}$ or ${ }^{133} \mathrm{Xe}$ radioactive tracers. Despite SPECT being more readily available and cheaper than PET, the latter is preferred in functional imaging studies due to its superior spatial and temporal resolution (86). Previous studies have demonstrated increased cerebral blood flow in the basal ganglia in patients with MHE, suggesting increased ammonia delivery to these areas, resulting in astrocytic dysfunction and cognitive alterations $(104,105)$. This is in agreement with a ${ }^{1} \mathrm{H}$ MRS study by Taylor-Robinson and colleagues that demonstrated Glx concentration was highest in the basal ganglia (82). However, SPECT studies that have investigated HE have been thwarted by small study sizes, limiting the conclusions that can presently be drawn from the use of this functional imaging tool.

\section{Summary}

In a patient with liver disease and neurological impairment, a CT scan and/or preferably, MRI of the brain can rule out other diagnoses and provide some corroborative evidence of HE. More researchbased modalities such as MRT, DTI, MRS, PET and SPECT have provided valuable insight into the pathogenesis of $\mathrm{HE}$, but have not been transformed into widely available diagnostic tools. Prior to this transformation, there must be consensus with regards to uniformity of study protocols, imaging sequences and analysis methodologies, both in MRI/MRS and in PET/SPECT, where promising functional data are emerging. In the future, a quantitative MR technique is most likely to give objective, reproducible and longitudinal diagnostic information in this common but under recognised complication of liver disease.

Acknowledgements: MJWM is supported by a Fellowship from the Wellcome Trust, London, United Kingdom. SDT-R holds grants from the British Medical Research Council, the Wellcome Trust, the national Institute of health Research of the United Kingdom (NIHR) and the Alan Morement Foundation (AMMF). All authors are grateful to the NIHR Biomedical Facility at Imperial College London for infrastructure support. 


\section{References}

(1) Mullen KD. Review of the final report of the 1998 Working Party on definition, nomenclature and diagnosis of hepatic encephalopathy. Aliment Pharmacol Ther 2007;25:11-16.

(2) Blei AT. Diagnosis and treatment of hepatic encephalopathy. Best Practice \& Research Clinical Gastroenterology 2000 12;14(6):959-974.

(3) Bajaj JS. Minimal hepatic encephalopathy is associated with motor vehicle crashes: the reality beyond the driving test. Hepatology 2009;50(4):1175.

(4) Bajaj JS. Minimal hepatic encephalopathy: a vehicle for accidents and traffic violations. Am J Gastroenterol 2007;102(9):1903.

(5) Prasad S. Lactulose improves cognitive functions and health-related quality of life in patients with cirrhosis who have minimal hepatic encephalopathy. Hepatology 2007;45(3):549.

(6) Lemberg A. Hepatic encephalopathy, ammonia, glutamate, glutamine and oxidative stress. Annals of Hepatology 2009;8(2):95.

(7) Häussinger D, Kircheis G, Fischer R, Schliess F, Dahl Sv. Hepatic encephalopathy in chronic liver disease: a clinical manifestation of astrocyte swelling and low-grade cerebral edema? J Hepatol 2000 6;32(6):1035-1038.

(8) Butterworth RF. Hepatic encephalopathy and brain edema in acute hepatic failure: Does glutamate play a role? Hepatology 1997;25(4):1032.

(9) Vaquero J. Brain edema in acute liver failure. A window to the pathogenesis of hepatic encephalopathy. Annals of Hepatology 2003;2(1):12.

(10) Jones EA. Is cerebral edema a component of the syndrome of hepatic encephalopathy? Hepatology 2002;35(5):1270.

(11) Häussinger D. Low grade cerebral edema and the pathogenesis of hepatic encephalopathy in cirrhosis. Hepatology 2006;43(6):1187.

(12) Quero Guillen JC. Diagnostic methods in hepatic encephalopathy. Clinica Chimica Acta 2006;365(1-2):1.

(13) Shah NJ, Neeb H, Kircheis G, Engels P, Häussinger D, Zilles K. Quantitative cerebral water content mapping in hepatic encephalopathy. Neuroimage 2008 7/1;41(3):706-717.

(14) Zeneroli ML. Globus pallidus alterations and brain atrophy in liver cirrhosis patients with encephalopathy: an MR imaging study. Magn Reson Imaging 1991;9(3):295.

(15) Pujol A. Hyperintensity in the globus pallidus on T1-weighted and inversion-recovery MRI: a possible marker of advanced liver disease. Neurology 1991;41(9):1526.

(16) Uchino A. Cerebral magnetic resonance imaging of liver cirrhosis patients. Clin Imaging 1994;18(2):123.

(17) Córdoba J. $1 \mathrm{H}$ magnetic resonance in the study of hepatic encephalopathy in humans. Metab Brain Dis 2002;17(4):415. 
(18) Butterworth RF. Manganese toxicity, dopaminergic dysfunction and hepatic encephalopathy. Metab Brain Dis 1995;10(4):259.

(19) Rose C. Manganese deposition in basal ganglia structures results from both portal-systemic shunting and liver dysfunction. Gastroenterology 1999;117(3):640.

(20) Spahr L. Increased blood manganese in cirrhotic patients: relationship to pallidal magnetic resonance signal hyperintensity and neurological symptoms. Hepatology 1996;24(5):1116.

(21) Katsuragi T. Cerebrospinal fluid manganese concentrations in patients with symmetric pallidal hyperintensities on T1 weighted MRI. Journal of Neurology, Neurosurgery and Psychiatry 1999;66(4):551.

(22) Pujol A. Hyperintense globus pallidus on T1-weighted MRI in cirrhotic patients is associated with severity of liver failure. Neurology 1993;43(1, Part 1):65.

(23) Binesh N, Huda A, Thomas MA, Wyckoff N, Bugbee M, Han S, et al. Hepatic encephalopathy: a neurochemical, neuroanatomical, and neuropsychological study. Journal of Applied Clinical Medical Physics 2006;7(1):86-96.

(24) Weissenborn K. Pallidal lesions in patients with liver cirrhosis: clinical and MRI evaluation. Metab Brain Dis 1995;10(3):219.

(25) Taylor Robinson SD. MR Imaging of the basal ganglia in chronic liver disease: Correlation of T 1weighted and magnetisation transfer contrast measurements with liver dysfunction and neuropsychiatric status. Metab Brain Dis 1995;10(2):175.

(26) Forton DM. Fatigue and primary biliary cirrhosis: association of globus pallidus magnetisation transfer ratio measurements with fatigue severity and blood manganese levels. Gut 2004;53(4):587.

(27) Fabiani G. Liver transplantion in a patient with rapid onset parkinsonism-dementia complex induced by manganism secondary to liver failure. Arq Neuropsiquiatr 2007;65(3A):685.

(28) Bastianello S. Fast spin-echo and fast fluid-attenuated inversion-recovery versus conventional spin-echo sequences for MR quantification of multiple sclerosis lesions. American journal of neuroradiology 1997;18(4):699.

(29) Rovaris M. Lesion load quantification on fast-FLAIR, rapid acquisition relaxation-enhanced, and gradient spin echo brain MRI scans from multiple sclerosis patients. Magn Reson Imaging 1999;17(8):1105.

(30) Englund E. Neuropathology of white matter lesions in vascular cognitive impairment. Cerebrovascular Diseases 2000;13(2):11.

(31) Minguez B. Decrease in the volume of white matter lesions with improvement of hepatic encephalopathy. American journal of neuroradiology 2007;28(8):1499.

(32) Rovira A. Decreased white matter lesion volume and improved cognitive function after liver transplantation. Hepatology 2007;46(5):1485.

(33) Rovira A. Normalization of T2 signal abnormalities in hemispheric white matter with liver transplant. Neurology 2002;59(3):335.

(34) Córdoba J. T2 hyperintensity along the cortico-spinal tract in cirrhosis relates to functional abnormalities. Hepatology 2003;38(4):1026. 
(35) Grover VPB. Current and future applications of magnetic resonance imaging and spectroscopy of the brain in hepatic encephalopathy. J Gastroenterol 2006;12(19):2969.

(36) Schaefer PW. Diffusion-weighted MR Imaging of the Brain. Radiology 2000;217(2):331.

(37) Kale RA. Demonstration of interstitial cerebral edema with diffusion tensor MR imaging in type C hepatic encephalopathy. Hepatology 2006;43(4):698.

(38) Sugimoto R. Value of the apparent diffusion coefficient for quantification of low-grade hepatic encephalopathy. Am J Gastroenterol 2008;103(6):1413.

(39) Lodi R. Diffusion MRI shows increased water apparent diffusion coefficient in the brains of cirrhotics. Neurology 2004;62(5):762.

(40) Miese F. 1H-MR spectroscopy, magnetization transfer, and diffusion-weighted imaging in alcoholic and nonalcoholic patients with cirrhosis with hepatic encephalopathy. American Journal of Neuroradiology 2006;27(5):1019.

(41) Kumar R. Voxel-based diffusion tensor magnetic resonance imaging evaluation of low-grade hepatic encephalopathy. Journal of Magnetic Resonance Imaging 2008;27(5):1061.

(42) Poveda MJ. Brain edema dynamics in patients with overt hepatic encephalopathy:: A magnetic resonance imaging study. Neuroimage 2010;52(2):481.

(43) Mardini $\mathrm{H}$. Magnetic resonance quantification of water and metabolites in the brain of cirrhotics following induced hyperammonaemia. J Hepatol 2010.

(44) Ziylan YZ, Uzm G, Bernard G, Diler AS, Bourre JM. Changes in the permeability of the bloodbrain barrier in acute hyperammonemia. Effect of dexamethasone. Molecular and Chemical Neuropathology 1993;20(3):203-218.

(45) Sobel RA. Glial Fibrillary Acidic Protein in Hepatic Encephalopathy An Immunohistochemical Study. J Neuropathol Exp Neurol 1981;40(6):625.

(46) Chen KC. Changes in brain cell shape create residual extracellular space volume and explain tortuosity behavior during osmotic challenge. Proc Natl Acad Sci U S A 2000;97(15):8306.

(47) McKinney AM. Acute hepatic encephalopathy: diffusion-weighted and fluid-attenuated inversion recovery findings, and correlation with plasma ammonia level and clinical outcome. American Journal of Neuroradiology 2010;31(8):1471.

(48) Nucifora PGP. Diffusion-Tensor MR Imaging and Tractography: Exploring Brain Microstructure and Connectivity1. Radiology 2007;245(2):367.

(49) Fridman V. MRI findings associated with acute liver failure. Neurology 2009;72(24):2130.

(50) Klauschen F, Goldman A, Barra V, Meyer-Lindenberg A, Lundervold A. Evaluation of automated brain MR image segmentation and volumetry methods. Hum Brain Mapp 2009;30(4):1310-1327.

(51) Smith SM. Accurate, robust, and automated longitudinal and cross-sectional brain change analysis. Neuroimage 2002;17(1):479.

(52) Smith SM. Normalized accurate measurement of longitudinal brain change. J Comput Assist Tomogr 2001;25(3):466.

(53) Ashburner J. Unified segmentation. Neuroimage 2005;26(3):839. 
(54) Dale AM. Cortical Surface-Based Analysis* $1:$ I. Segmentation and Surface Reconstruction. Neuroimage 1999;9(2):179.

(55) Fischl B. Cortical Surface-Based Analysis* 1:: II: Inflation, Flattening, and a Surface-Based Coordinate System. Neuroimage 1999;9(2):195.

(56) Jenkinson M, Smith S. A global optimisation method for robust affine registration of brain images. Med Image Anal 2001 6;5(2):143-156.

(57) Kakeda S. The efficacy of a voxel-based morphometry on the analysis of imaging in schizophrenia, temporal lobe epilepsy, and Alzheimer's disease/mild cognitive impairment: a review. Neuroradiology 2010;52(8):711.

(58) Patel N. Changes in brain size in hepatic encephalopathy: a coregistered MRI study. Metab Brain Dis 2004;19(3):431.

(59) GarciaMartinez R. Hepatic encephalopathy is associated with posttransplant cognitive function and brain volume. Liver Transplantation 2011;17(1):38.

(60) Guevara M. Cerebral magnetic resonance imaging reveals marked abnormalities of brain tissue density in patients with cirrhosis without overt hepatic encephalopathy. J Hepatol 2010.

(61) Pagani E. Basic concepts of advanced MRI techniques. Neurological Sciences 2008;29:290.

(62) Wolff SD. Magnetization transfer imaging: practical aspects and clinical applications. Radiology 1994;192(3):593.

(63) Rovira A. MR imaging findings in hepatic encephalopathy. AJNR, American Journal of Neuroradiology 2008;29(9):1612.

(64) Rovira A, Grive E, Pedraza S, Rovira A, Alonso J. Magnetization Transfer Ratio Values and Proton MR Spectroscopy of Normal-appearing Cerebral White Matter in Patients with Liver Cirrhosis. Am J Neuroradiol 2001 June 1;22(6):1137-1142.

(65) Miese FR. Voxel-based analyses of magnetization transfer imaging of the brain in hepatic encephalopathy. World Journal of Gastroenterology 2009;15(41):5157.

(66) Goel A. Cerebral oedema in minimal hepatic encephalopathy due to extrahepatic portal venous obstruction. Liver international 2010;30(8):1143.

(67) Córdoba J, Alonso J, Rovira A, Jacas C, Sanpedro F, Castells L, et al. The development of lowgrade cerebral edema in cirrhosis is supported by the evolution of $1 \mathrm{H}$-magnetic resonance abnormalities after liver transplantation. J Hepatol 2001 11;35(5):598-604.

(68) Schweinsburg BC, Taylor MJ, Alhassoon OM, Videen JS, Brown GG, Patterson TL, et al. Chemical Pathology in Brain White Matter of Recently Detoxified Alcoholics: A 1H Magnetic Resonance Spectroscopy Investigation of Alcohol-Associated Frontal Lobe Injury. Alcoholism: Clinical and Experimental Research 2001;25(6):924-934.

(69) Howseman AM. Functional magnetic resonance imaging: imaging techniques and contrast mechanisms. Philosophical transactions - Royal Society.Biological sciences 1999;354(1387):1179.

(70) Zafiris O. Neural mechanism underlying impaired visual judgement in the dysmetabolic brain: an fMRI study. Neuroimage 2004;22(2):541. 
(71) Zhang LJ. Neural mechanism of cognitive control impairment in patients with hepatic cirrhosis: a functional magnetic resonance imaging study. Acta Radiol 2007;48(5):577.

(72) MacLeod CM. Half a century of research on the Stroop effect: An integrative review. Psychol Bull 1991;109(2):163.

(73) Power JD. The development of human functional brain networks. Neuron 2010;67(5):735.

(74) Hafkemeijer A. Imaging the default mode network in aging and dementia. Biochimica et Biophysica Acta: Molecular basis of disease 2011.

(75) Broyd SJ. Default-mode brain dysfunction in mental disorders: a systematic review. Neuroscience Biobehavioral Reviews 2009;33(3):279.

(76) McPhail MJW. The role of magnetic resonance imaging and spectroscopy in hepatic encephalopathy. Metab Brain Dis 2010;25(1):65.

(77) TaylorRobinson SD. Cerebral proton and phosphorus-31 magnetic resonance spectroscopy in patients with subclinical hepatic encephalopathy. Metab Brain Dis 1999;19(5):389.

(78) Patel N. Intracellular pH measurements of the whole head and the basal ganglia in chronic liver disease: a phosphorus-31 MR spectroscopy study. Metab Brain Dis 2000;15(3):223.

(79) Hamilton G. Changes in brain intracellular pH and membrane phospholipids on oxygen therapy in hypoxic patients with chronic obstructive pulmonary disease. Metab Brain Dis 2003;18(1):95.

(80) Hamilton G, Patel N, Forton DM, Hajnal JV, Taylor-Robinson SD. Prior knowledge for time domain quantification of in vivo brain or liver 31P MR spectra. NMR Biomed 2003;16(3):168-176.

(81) Cox IJ. Development and applications of in vivo clinical magnetic resonance spectroscopy. Prog Biophys Mol Biol 1996;65(1-2):45.

(82) Taylor Robinson SD. Regional variations in cerebral proton spectroscopy in patients with chronic hepatic encephalopathy. Metab Brain Dis 1994;9(4):347.

(83) Singhal A. Two-dimensional MR spectroscopy of minimal hepatic encephalopathy and neuropsychological correlates in vivo. Journal of Magnetic Resonance Imaging 2010;32(1):35.

(84) TaylorRobinson SD. Cerebral Phosphorus-31 magnetic resonance spectroscopy in patients with chronic hepatic encephalopathy. Hepatology 1994;20(5):1173.

(85) Taylor Robinson SD. MR imaging and spectroscopy of the basal ganglia in chronic liver disease: correlation of T 1-weighted contrast measurements with abnormalities in proton and phosphorus-31 MR spectra. Metab Brain Dis 1996;11(3):249.

(86) Stewart C, Reivich M, Lucey M, Gores G. Neuroimaging in hepatic encephalopathy. Clinical Gastroenterology and Hepatology 2005;3(3):197-207.

(87) Miller PW. Synthesis of $11 \mathrm{C}, 18 \mathrm{~F}, 15 \mathrm{O}$, and $13 \mathrm{~N}$ radiolabels for positron emission tomography. Angewandte Chemie (International ed.in English) 2008;47(47):8998.

(88) Lockwood $\mathrm{AH}$. Altered cerebral blood flow and glucose metabolism in patients with liver disease and minimal encephalopathy. Journal of Cerebral Blood Flow and Metabolism 1991;11(2):331.

(89) Lockwood AH. Correlations between cerebral glucose metabolism and neuropsychological test performance in nonalcoholic cirrhotics. Metab Brain Dis 2002;17(1):29. 
(90) Ahl B, Weissenborn K, van den Hoff J, Fischer-Wasels D, Köstler H, Hecker H, et al. Regional differences in cerebral blood flow and cerebral ammonia metabolism in patients with cirrhosis. Hepatology 2004;40(1):73-79.

(91) Lockwood AH. The dynamics of ammonia metabolism in man. Effects of liver disease and hyperammonemia. J Clin Invest 1979;63(3):449.

(92) Lockwood AH. Blood-brain barrier to ammonia in humans. Journal of Cerebral Blood Flow and Metabolism 1984;4(4):516.

(93) Lockwood AH. Cerebral ammonia metabolism in patients with severe liver disease and minimal hepatic encephalopathy. Journal of Cerebral Blood Flow and Metabolism 1991;11(2):337.

(94) Keiding S. Brain metabolism of $13 \mathrm{~N}$-ammonia during acute hepatic encephalopathy in cirrhosis measured by positron emission tomography. Hepatology 2006;43(1):42.

(95) Berding G. Radiotracer imaging studies in hepatic encephalopathy: ISHEN practice guidelines. Liver International 2009;29(5):621.

(96) Macdonald GA. Cerebral benzodiazepine receptor binding in vivo in patients with recurrent hepatic encephalopathy. Hepatology 1997;26(2):277.

(97) Jalan R. Increased availability of central benzodiazepine receptors in patients with chronic hepatic encephalopathy and alcohol related cirrhosis. Gut 2000;46(4):546.

(98) Lozeva Thomas V. Serotonin brain circuits with a focus on hepatic encephalopathy. Metab Brain Dis 2004;19(3):413.

(99) Weissenborn K. Altered striatal dopamine D 2 receptor density and dopamine transport in a patient with hepatic encephalopathy. Metab Brain Dis 2000;15(3):173.

(100) Cagnin A. In vivo imaging of cerebral "peripheral benzodiazepine binding sites" in patients with hepatic encephalopathy. Gut 2006;55(4):547.

(101) Desjardins P. The "peripheral-type" benzodiazepine (omega 3) receptor in hyperammonemic disorders. Neurochem Int 2002;41(2-3):109.

(102) Iversen P. Peripheral benzodiazepine receptors in the brain of cirrhosis patients with manifest hepatic encephalopathy. Eur J Nucl Med 2006;33(7):810.

(103) Levin CS. Primer on molecular imaging technology. Eur J Nucl Med 2005;32:325.

(104) O'Carroll RE. Regional cerebral blood flow and cognitive function in patients with chronic liver disease. The Lancet 1991;337(8752):1250.

(105) Catafau AM. Relationship between cerebral perfusion in frontal-limbic-basal ganglia circuits and neuropsychologic impairment in patients with subclinical hepatic encephalopathy. The Journal of Nuclear Medicine 2000;41(3):405. 


\begin{tabular}{llll}
\hline $\begin{array}{l}\text { Imaging } \\
\text { technique }\end{array}$ & Availability & Findings in Hepatic Encephalopathy & $\begin{array}{l}\text { Recommended in } \\
\text { the clinic }\end{array}$ \\
\hline CT Brain & Standard & $\begin{array}{l}\text { Cerebral edema in ALF } \\
\text { Rules out some common differential } \\
\text { diagnoses }\end{array}$ & Yes \\
& & & \\
& &
\end{tabular}

\section{MRI brain}

\begin{tabular}{|c|c|c|c|}
\hline $\mathrm{T}_{1}$-weighted & Standard & $\begin{array}{l}\text { Basal ganglia hypersensitivity } \\
\text { (not HE specific) } \\
\text { Cerebral oedema in ALF } \\
\text { Quantitative brain volume by } \\
\text { research statistical methods }\end{array}$ & Yes (not in ALF) \\
\hline \multicolumn{4}{|l|}{$T_{2}$-weighted } \\
\hline FLAIR & Standard & $\begin{array}{l}\text { White matter lesions in cases of low } \\
\text { grade HE }\end{array}$ & Yes (not in ALF) \\
\hline DWI & Standard & $\begin{array}{l}\text { High ADC in CHE } \\
\text { Low ADC in ALF }\end{array}$ & \\
\hline $\begin{array}{l}\text { Diffusion tensor } \\
\text { imaging }\end{array}$ & Research & $\begin{array}{l}\text { Increased mean diffusivity in } \\
\text { corticospinal tracts }\end{array}$ & No \\
\hline $\begin{array}{l}\text { Magnetisation } \\
\text { Transfer }\end{array}$ & $\begin{array}{l}\text { Available in } \\
\text { specialist centres }\end{array}$ & Low MTR in white matter & No \\
\hline $\begin{array}{l}{ }^{1} \mathrm{H} \text { MR } \\
\text { Spectroscopy }\end{array}$ & $\begin{array}{l}\text { Available in } \\
\text { specialist centres }\end{array}$ & $\begin{array}{l}\text { High Glx (glutamine/glutamate) } \\
\text { Low myoinositol and choline in basal } \\
\text { ganglia and frontal white matter }\end{array}$ & No \\
\hline Functional MRI & Research & $\begin{array}{l}\text { Deactivation of the default mode } \\
\text { network including the anterior } \\
\text { cingulate }\end{array}$ & \\
\hline \multicolumn{4}{|l|}{$\begin{array}{l}\text { Nuclear } \\
\text { medicine }\end{array}$} \\
\hline PET & Research in $\mathrm{HE}$ & $\begin{array}{l}\text { Reduced glucose uptake in the } \\
\text { anterior cingulate }\end{array}$ & No \\
\hline SPECT & Research in $\mathrm{HE}$ & $\begin{array}{l}\text { Increased blood flow to the basal } \\
\text { ganglia }\end{array}$ & No \\
\hline
\end{tabular}

Table 1: Approach to different imaging modalities in HE in clinical and investigative use 
- CT Brain can demonstrate edema in ALF and assists in the differential diagnosis of neurological impairment in cirrhosis

- MR Brain is not recommended routinely in ALF but can assist in the diagnosis of HE in cirrhosis where it is the preferred method of brain imaging

- Quantification of cerebral metabolites or brain water is possible but not yet widely used diagnostically

- $\quad$ PET/SPECT are powerful but expensive research tools

Box 1: Clinical and research imaging techniques applied in the diagnosis of hepatic encephalopathy 


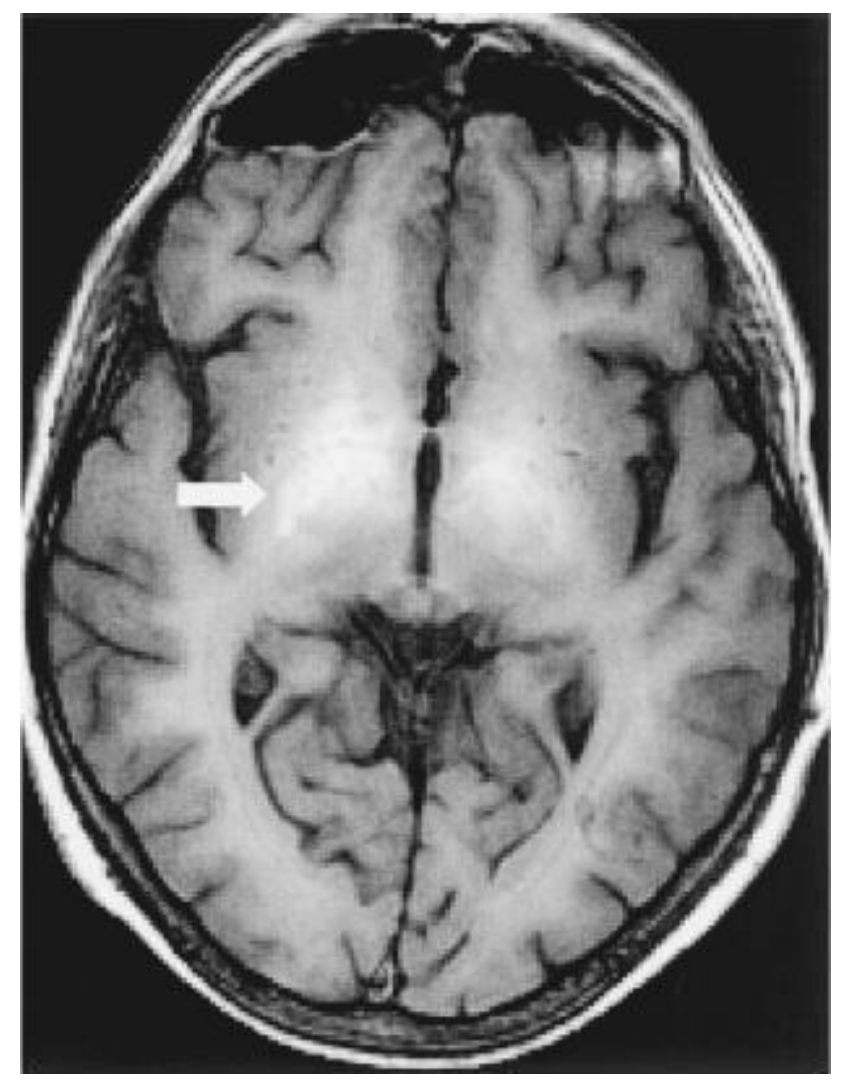

Figure 1: $T_{1}$-weighted magnetic resonance image of the brain of a patient with cirrhosis. The arrow demonstrates the area of pallidal hyperintensity. Reproduced from Córdoba J, et al. ${ }^{1} \mathrm{H}$ magnetic resonance in the study of hepatic encephalopathy in humans. Metab Brain Dis 2002;17(4):415. With permission from Springer Inc.

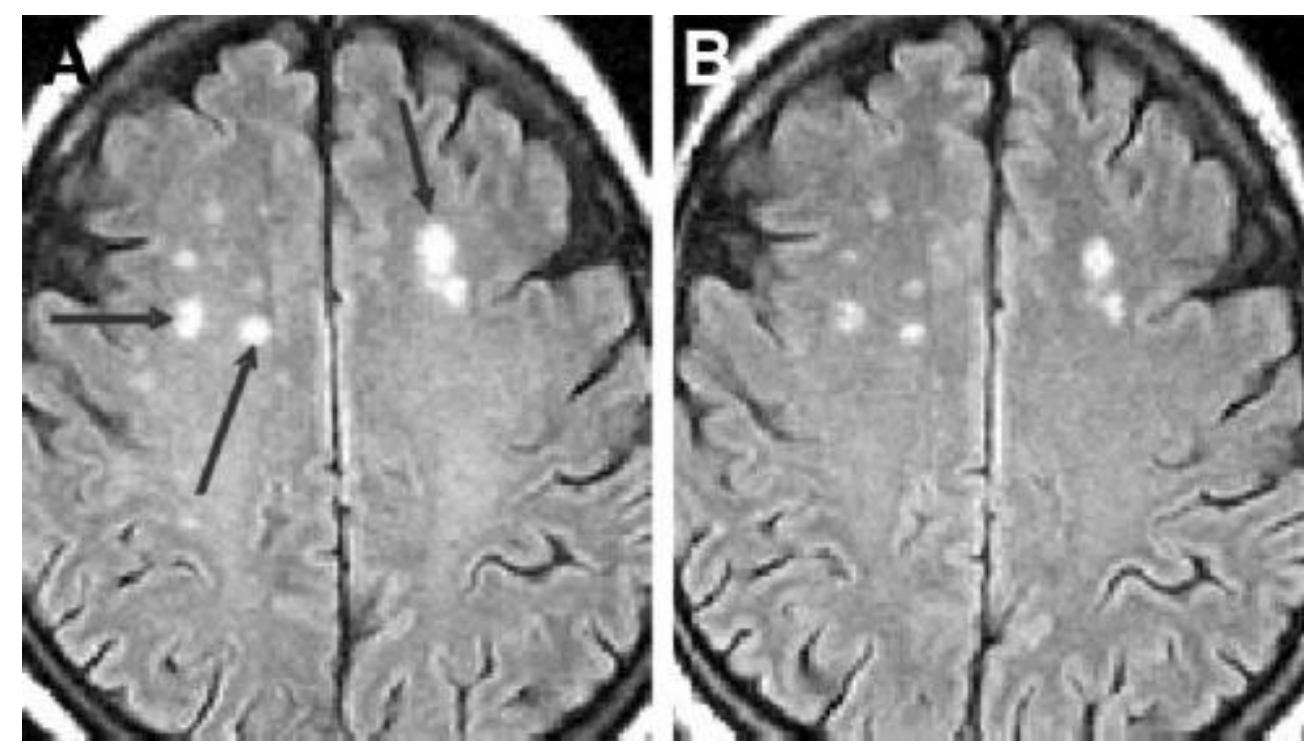


Figure 2: A. Baseline fast-FLAIR MRI image of a hepatitis $C$ patients prior to liver transplantation. Focal lesions can be visualized in the subcortical white matter (arrows). B. Same MRI study 6 months post-liver transplant in the same patient; there is a noticeable decrease in the size of focal white matter lesions. Reproduced from Rovira A, et al. Decreased white matter lesion volume and improved cognitive function after liver transplantation. Hepatology 2007;46(5):1485. With permission from John Wiley and Sons Inc.

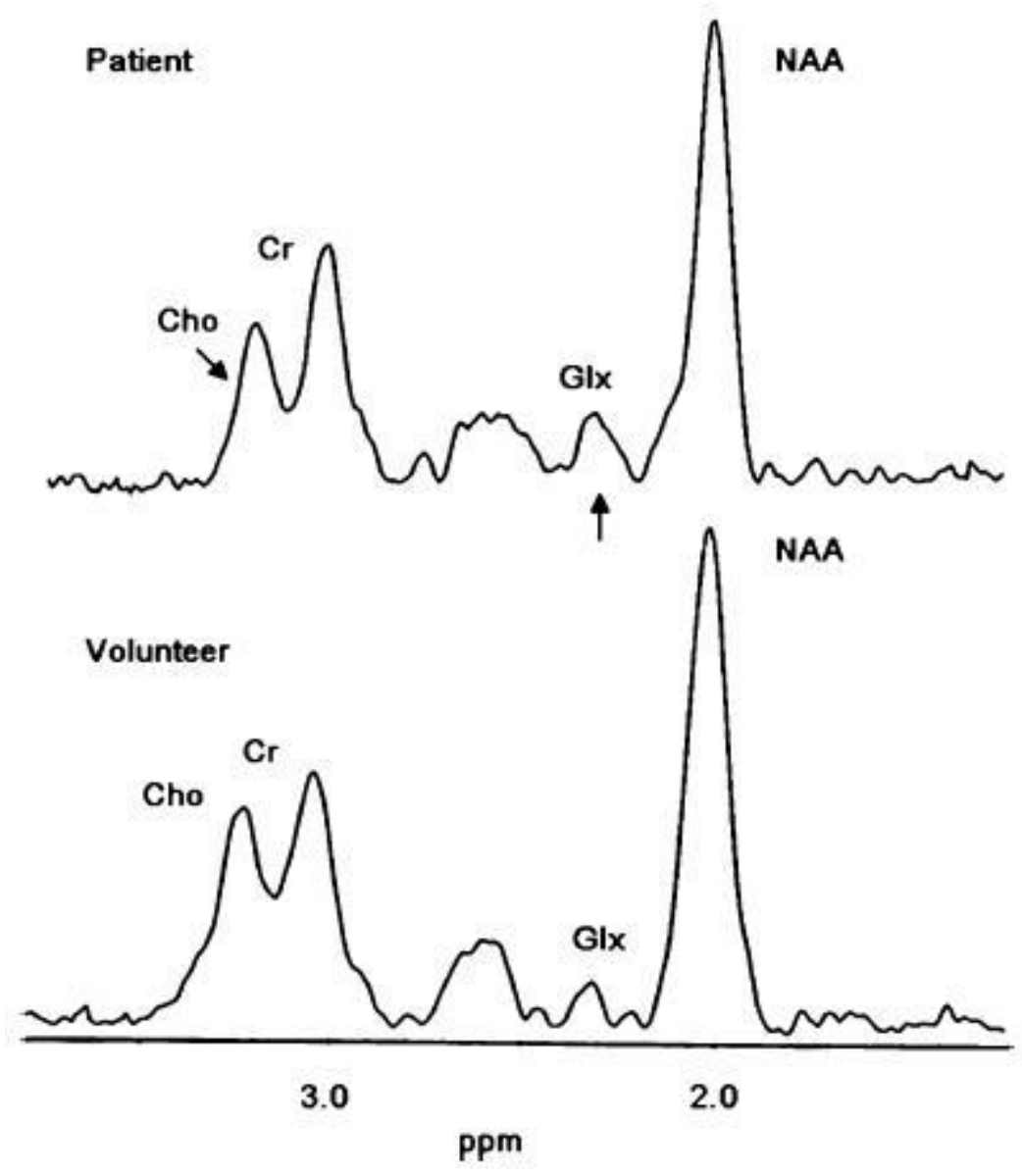

Figure 3: ${ }^{1} \mathrm{H}$ MR spectra from the basal ganglia of a patient with chronic hepatic encephalopathy and healthy volunteer, demonstrating decreased choline/creatine ratio and increased glutamate/glutamine resonance in the CHE patient. Cho: choline; Cr: creatine; Glx: glutamine/glutamate; NAA: N-acetyl aspartate. Reproduced from Taylor-Robinson SD, et al. Regional variations in cerebral proton spectroscopy in patients with chronic hepatic encephalopathy. Metab Brain Dis. 1994 Dec;9(4):347-59 With permission from Springer Inc. 


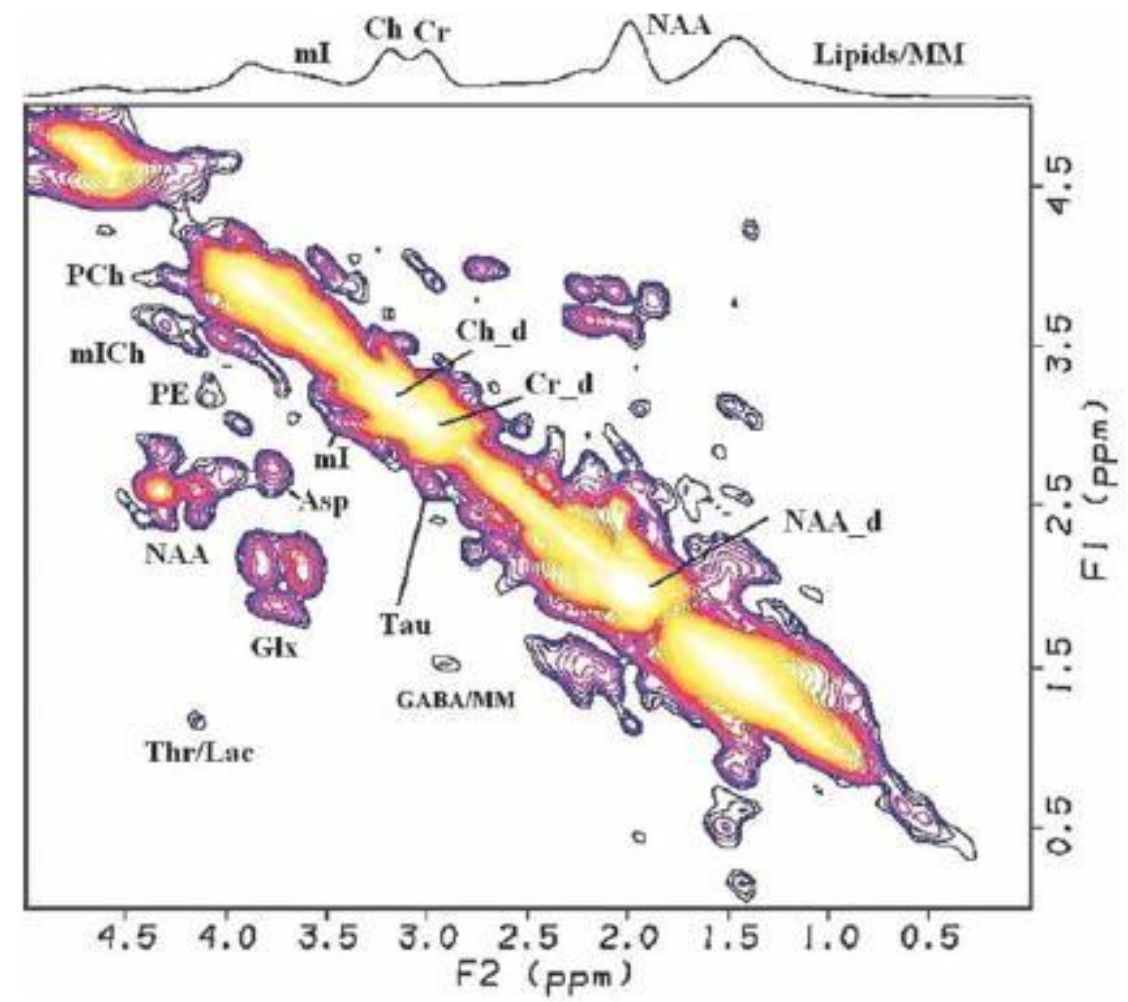

Figure 4: The 2D-COSY spectrum from the occipital lobe of a 51-year-old patient with minimal hepatic encephalopathy. Cr: creatine; Ch: choline; Glx: glutamate/glutamine; NAA: N-acetyl aspartate; Asp: aspartate; PE: phosphoethanolamine; PCh: phosphocholine; ml: myoinositol; Tau: taurine; ThrLac: overlapping cross peaks of threonine and lactate; GABA: gaba-aminobutyric acid; mICh: overlapping cross peaks of myo-inositol and choline; MM: macromolecules. Reproduced from: Singhal A et al. Two-dimensional MR spectroscopy of minimal hepatic encephalopathy and neuropsychological correlates in vivo. Journal of magnetic resonance imaging 2010;32(1):35. With permission from John Wiley and Sons Inc. 


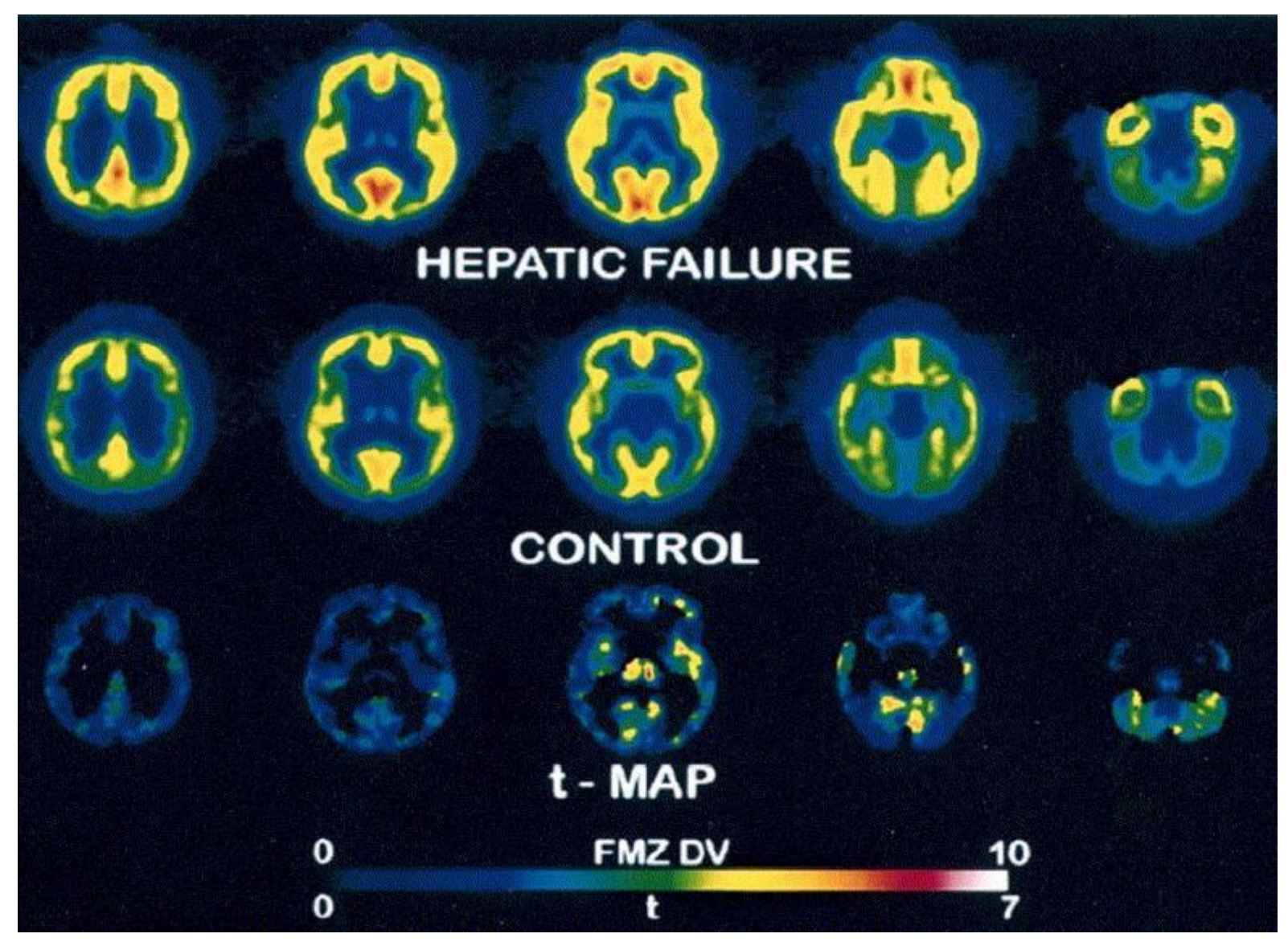

Fi Figure 5: Analyses of benzodiazepine binding in patients with hepatic failure (top row), control (middle row), and voxel-by-voxel comparison of between-group differences (bottom row). The greatest changes in distribution volume of flumazenil was seen in the cerebellum. Reproduced from MacDonald GA, et al. Cerebral benzodiazepine receptor binding in vivo in patients with recurrent hepatic encephalopathy. Hepatology 1997;26(2):277. With permission from John Wiley and Sons Inc. gure 5 